\title{
Comparative study of two methods for Handwritten Devanagari Numeral Recognition
}

\author{
S.L.Mhetre ${ }^{1}$, Prof.M.M.Patil ${ }^{2}$ \\ '(E\&TC Dept,SAE/ Pune University,India) \\ ${ }^{2}$ (E\&TC Dept,SAE/Pune University,India)
}

\begin{abstract}
In this paper two different methods for Numeral Recognition are proposed and their results are compared. The objective of this paper is to provide an efficient and reliable method for recognition of handwritten numerals. First method employs Grid based feature extraction and recognition algorithm. In this method the features of the image are extracted by using grid technique and this feature set is then compared with the feature set of database image for classification. While second method contains Image Centroid Zone and Zone Centroid Zone algorithms for feature extraction and the features are applied to Artificial Neural Network for recognition of input image. Machine text recognition is important research area because of its applications in many areas like Bank, Post office, Hospitals etc.
\end{abstract}

Keywords: Handwritten Numeral Recognition, Grid Technique, ANN, Feature Extraction, Classification.

\section{INTRODUCTION}

Lot of research has been done on printed and handwritten character/numeral recognition. Between these two the handwritten text recognition has low recognition rate as compared to printed text due to number of challenges arising while processing them. So we can conclude that the handwritten text recognition is still in development stage. Whereas printed text recognition has reached to exploitation level. Again Devanagari script is the script used by more than 300 million people for documentation. Many official languages in India like Hindi, Marathi, Sindhi, Sanskrit are the form of Devanagari script. Among which Hindi is the third most popular language in world. The Devanagari OCR thus will be helpful in digitizing the ancient literatures, books, newspapers etc. and can also be used for official purposes where computers (machines) are used wildly [1]. Thus here we have proposed algorithms for recognition of Handwritten Devanagari Numeral.

The recognition can be either on-line or offline recognition. Various methods has been discussed for on-line and off-line character recognition by R. Plamondon and S.N. Srihari [2]. Off-line recognition is of two types : Printed text recognition and Handwritten text recognition. A good survey has been made by R. Jayadevan, Satish R. Kolhe, Pradeep M. Patil, and Umapada Pal on off-line Devanagari recognition which will be very useful for the researchers working in this field [3].Though large research papers have been published on Devanagari Numeral Recognition but the first paper was reported in 1977, which uses structural approach for recognition [4]. A wavelet filter-based multi resolution analysis is carried out on input images and MLP classifier is used for recognition of numerals by Chaudhuri and Bhattacharya [5]. Bajaj, Dey used median filter to remove noise in input image[6]. For recognition Bajaj, Dey used features like descriptive features, density features and moment features of right, left, upper and lower profile curves and finally combined the decisions of multiple classifiers [6]. While box approach is used by Hanmandlu for feature extraction [7]. Thinning based features are used by Elnagar and Harous [8]. From a thinned image they extracted three different types of feature types like branch, end and cross points by representing them syntactically.

\section{DATA Collection}

Here database for Devanagari numerals is created having 1000 samples written from 10 different individuals of different age group. They were asked to write numerals in devanagari from $0-9$ on a A4 size paper. Handwriting in general can be categorize as : Cursive or hand printed hand writing. The numerals in Devanagari are written in hand printed handwriting form i.e. the numerals are not connected to each other like in cursive. The sample for Devanagari Numerals is as shown in fig below:

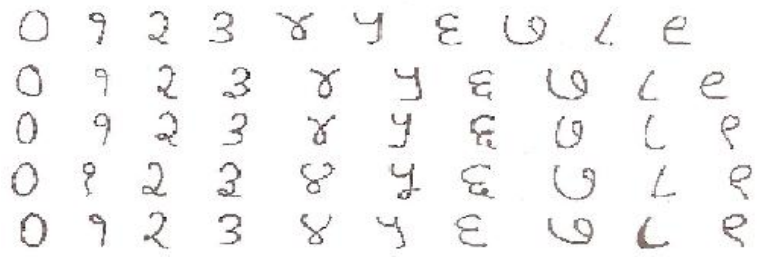

figure 1. Devanagari Numerals 


\section{PRE-PROCESSING}

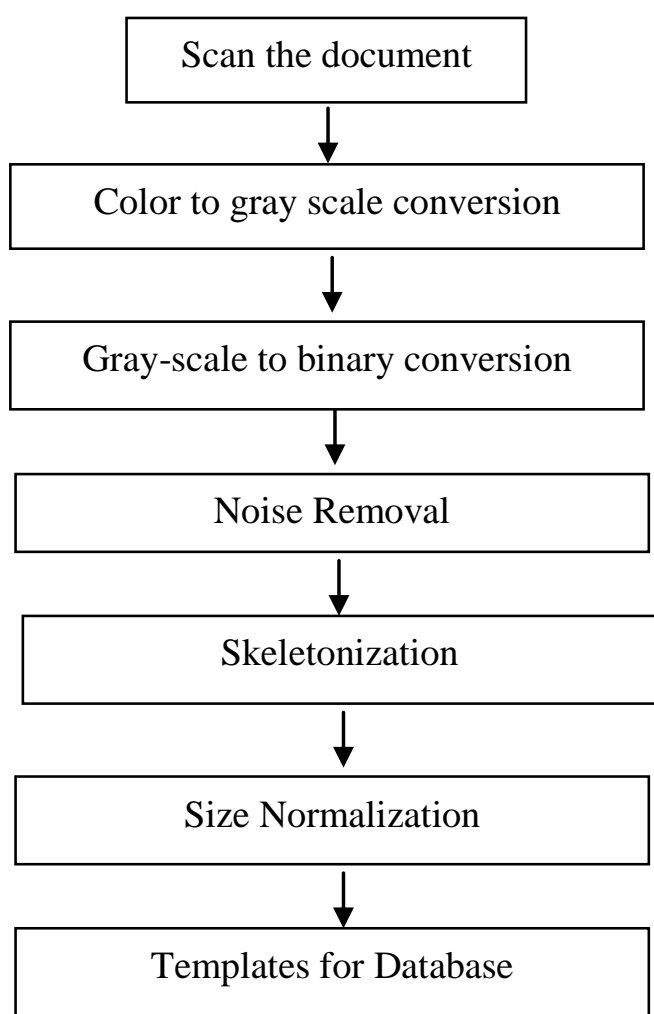

Numerical recognition is nothing but the conversion of handwritten or typed numerical document into machine understandable form. For this the document is first scanned using a regular scanner. Before performing the feature extraction directly, first the scanned data/image is passed through some pre-processing steps (shown in the flow chart below), these steps are as follows:

- If the scanned document is a color image it is converted into a gray scale image. The conversion is carried out by removing the hue and saturation while retaining the luminance.

- The gray scale image is still a raw image; it may carry some unwanted information. This information is termed as noise. The noise/distortion may introduce while scanning the image. The noise is of various types like salt and pepper noise, shot noise etc. Median filter is used to remove such noise.

- After filtering the image it is converted into binary form. The process is also called as Image Segmentation. Image segmentation is performed by assigning a variable to a threshold value, if the value of pixel in gray scale image is equal or above the threshold value then the pixel is replaced by 1 else 0 . At the end the image is inverted for easy processing.

- The image obtained after binarization is sent for thinning / skeletonization process. Skeletonization reduces the width of numerals from many pixels to one.

- Finally the image is restored to a standard dimension 128*96. (It is not necessary to use/take these dimensions only. Our aim is to create a database with images having fixed size. Any dimensions can be taken to achieve feasibility in operation.)

\section{FEATURE EXTRACTION}

This is the most important step in character/numeral recognition, since classification is done on the basis of the set of features extracted during this step. Feature extraction simply means the acquisition or measurement of those parameters of input image that are most useful for classification purpose. Many of such features are invented and used by scientists for pattern classification.

In this paper we are using two different feature extraction methods. Features can be mainly classified into two different types: Structural Features and Numerical Features. Among which structural features are related to the shape or structure of input image (easy for human being to understand), while numerical features are concerned with numeric values (distance, height, width etc) extracted from the input image. These numeric values can be real or binary. Humans cannot conclude anything from this information. In first method we are extracting the structural features of input image. For this Grid is formed on the input image and the image is resized to $8 * 6$ block and $16 * 12$ block size, while second method uses distance based features i.e. numerical features of input image. Both the methods are explained as below: 


\subsection{Grid Based Method}

1) Grid based feature extraction is a type of pixel oriented feature extraction. The steps for this method are as follows:

2) After pre-processing the sizes of all the images are normalized to $x^{*} y$ pixels. Size normalization is a must condition to obtain best results for character/ numeral recognition. A grid is formed on the image of size $a * b$ such that $a<<x$ and $b<<y$. In this way the input image segmented into number of rectangular cells.

3) Next step is to find out the rectangular cells (grid) that contains numeral contents. For such grid calculate the number of black pixels.

4) Now create a matrix of size $a * b$ having same size of grid. Here our assumption is that each element (pixel) of newly created matrix $a * b$ belongs to each grid of the image.

5) The value of pixel in the matrix will be 1 if the number of black pixels in the corresponding grid is more than 3 else 0 .

6) The procedure is repeated for each pixel in the new matrix.

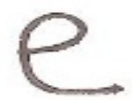

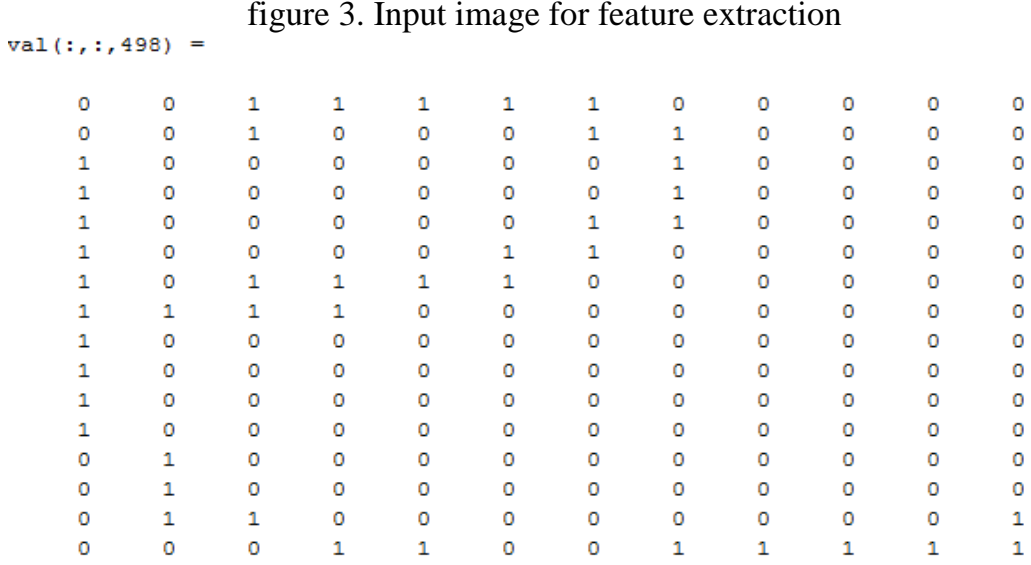

figure 4. Grid features of Devanagari Numeral

\subsection{ICZ \& ZCZ}

\subsubsection{Image Centroid Zone (ICZ)}

Algorithm:

7) Calculate centroid of input image.

8) Divide the image into equal zones.

9) After zone formation, calculate the distance between centroid and each numeral pixel in the zone.

10) Now calculate the average distance corresponding to that zone.

11) Repeat procedure 3) and 4) for each zone.

12) In this way we obtain the number of features equal to the number of zones created in second step.

\subsubsection{Zone Centroid Zone (ZCZ)}

\section{Algorithm:}

13) Divide the image into equal zones.

14) Calculate centroid for each zone.

15) After zone formation, calculate the distance between the zone centroid and each numeral pixel in the zone.

16) Now calculate the average distance corresponding to that zone.

17) Repeat procedure 3) and 4) for each zone.

18) In this way we obtain the number of features equal to the number of zones created in first step.

\section{Classification}

Classification stage requires the features of the template images extracted in the previous stage of feature extraction. 


\subsection{Matching Score}

The recognition by matching score is performed by comparing the grid feature of the input image with all the template images in the database. The template image for which we will get minimum matching score; that image will be the recognized numeral. For this first the input image (to be recognized) is scanned using an optical scanner and the scanned document is passed through various pre-processing steps shown in the fig.(2).

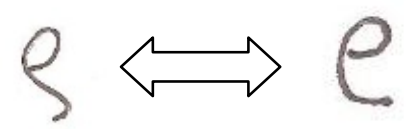

figure 5. Input image matched with template image

As shown in fig above, first image is the input image which is matched with the second template image in the database with minimum dist 39 shown in fig below.

\begin{tabular}{|c|c|c|c|c|c|c|c|c|c|}
\hline \multicolumn{10}{|c|}{$\boxplus$ dist <1×500 double> } \\
\hline & & 455 & 456 & 457 & $45 \&$ & 459 & 460 & 461 & 462 \\
\hline 1 & 53 & 69 & 49 & 40 & 39 & 56 & 66 & 68 & \\
\hline
\end{tabular}

Figure 6. Minimum distance (or Matching Score)

\subsection{Artificial Neural Network}

The success rate of Artificial Neural Network is highly dependent on their efficiency of accurate classification of the input (images) also called as recognition rate. Character/ Numeral recognition is the most important application of ANN(Artificial Neural Network). The model for Artificial Neural Network is shown in fig. below:

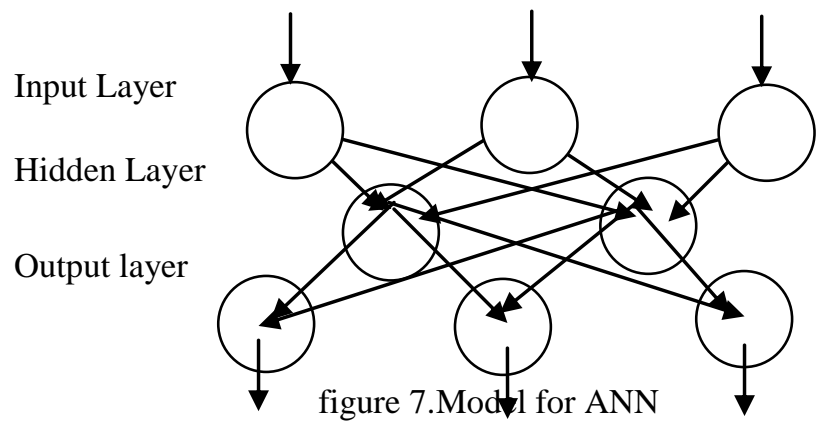

Scientists(specially brain modellers) were inspired by human nervous system and they develop a model of ANN. ANN uses numerical features for classification of the input image.

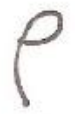

figure 8.input image for ICZ,ZCZ feature extraction

Numeral shown in fig. is first scanned and then passed through different pre processing steps. Then its features are extracted using ICZ \& ZCZ feature extraction technique. The feature set for the input image is shown in fig. below:

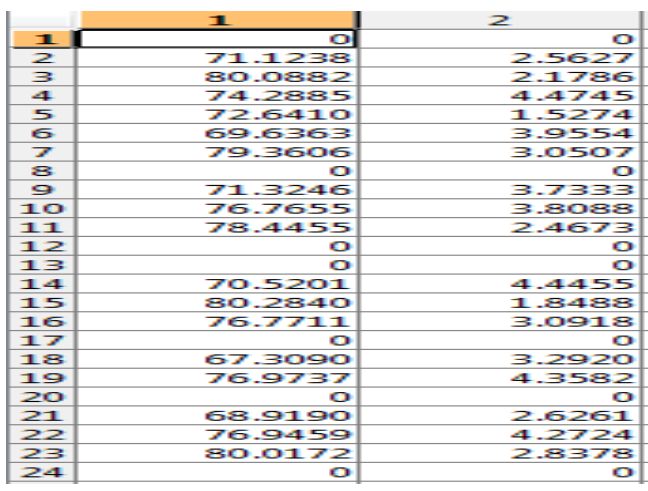

figure 9. ICZ,ZCZ Feature set 


\section{COMPARISON OF RESUlT}

As explained in the paper, first method uses Grid based technique for feature extraction and then matching score is calculated from the extracted features for classification. In second method the feature set which is extracted using ICZ \& ZCZ feature extraction technique is applied to Artificial Neural Network for classification. The database used for both the techniques is same. Also the images used for testing both the system are same, so that we can easily compare the result obtained from both the methods.

Table 1. Comparison of Results

\begin{tabular}{|l|l|l|l|l|l|}
\hline Sr.No. & Method & Template image & Test image & Correct recognition & Result $(\%)$ \\
\hline 1 & Grid Technique & 500 & 500 & 418 & 83.60 \\
\hline 2 & ANN & 500 & 500 & 432 & 86.40 \\
\hline
\end{tabular}

\section{CONCLUSION}

This paper aims at recognition of devanagari numerals using grid based and Artificial Neural Network Approach. The result of ANN is good as compared to Grid Technique. The result of Neural Network depends on how much it is trained. If the the number of samples provided for training is increased then definitely we can achieve results upto $96 \%$. The methods used here for numeral recognition can also be used for printed or handwritten Devanagari character recognition. Also we can use the Grid technique for offline signature verification purpose.

\section{REFERENCES}

[1] U. Bhattacharya and B. B. Chaudhuri, "Handwritten numeral databases of Indian scripts and multistage recognition of mixed numerals," IEEE Trans. Pattern Anal. Mach. Intell., vol. 31, no. 3, pp. 444-457, Mar. 2009.

[2] R. Plamondon and S.N. Srihari., 2000, "Online and off-line handwriting recognition: a comprehensive survey.", Pattern Analysis and Machine Intelligence, IEEE Transac-tions on, vol.22(1), pp 63-84.

[3] R. Jayadevan, Satish R. Kolhe, Pradeep M. Patil, and Umapada Pal, "Offline Recognition of Devanagari Script: A Survey," IEEE Transactions on Systems, man, and Cybernetics-part c: Applications and Reviews, vol. 41, no. 6, November 2011.

[4] I. K. Sethi and B. Chatterjee, "Machine recognition of hand printed Devanagari numerals," J. Inst. Electron. Telecommun. Eng., vol. 22, pp. 532-535, 1977 .

[5] U. Bhattacharya and B. B. Chaudhuri, "Handwritten numeral databases of Indian scripts and multistage recognition of mixed numerals," IEEE Trans. Pattern Anal. Mach. Intell., vol. 31, no. 3, pp. 444-457,Mar. 2009.

[6] R. Bajaj, L. Dey, and S. Chaudhuri, "Devanagari numeral recognition by combining decision of multiple connectionist classifiers," Sadhana, vol. 27, no. 1, pp. 59-72, 2002.

[7] M. Hanmandlu, J. Grover, V. K. Madasu, and S. Vasikarla, "Input fuzzy modeling for the recognition of handwritten Hindi numerals," in Proc. Int. Conf. Inf. Technol., 2007, pp. 208-213.

[8] A. Elnagar and S. Harous, "Recognition of handwritten Hindi numerals using structural descriptors," J. Exp. Theor. Artif. Intell., vol. 15, no. 3,pp. 299-214, 2003. 